\title{
A FAMILY OF NEWFORMS
}

\author{
R. A. RANKIN
}

\section{Introduction}

Bounds for sums of powers of cusp form coefficients were recently obtained in [10]. Subsequently, Moreno and Shahidi [4] have shown that, in the case of Ramanujan's function $\tau(n)$, more precise estimates can be obtained for the fourth power. They have established that the Dirichlet series

$$
\sum_{n=1}^{\infty} \tau^{4}(n) n^{-s}
$$

has a double pole at $s=13 / 2$ and so have deduced that

$$
\sum_{n \leqq x} \tau_{0}^{4}(n) \sim A x \log x
$$

as $x \rightarrow \infty$, where $A$ is a positive constant and

$$
\tau_{0}(n)=\tau(n) n^{-11 / 2} .
$$

This improves the estimates

$$
x \ll \sum_{n \leqq x} \tau_{0}^{4}(n) \ll x \log ^{3} x
$$

found in [10]. Presumably, asymptotic results of the form (1.1) hold also for fourth powers of other newform coefficients not of complex multiplication type.

It is the purpose of the present paper to study an infinite family of newforms of different weights for which precise asymptotic formulae can be found for every sum of even powers, and which do not obey an asymptotic formula of the form (1.1) for the fourth power sum of the coefficients. As indicated at the end of [10] these forms are associated with Grössencharacters belonging to imaginary quadratic fields and, for illustration, we take the simplest case, where the field is the field of Gaussian integers $K=\boldsymbol{Q}(i)$.

For each $\xi \in K$ we put

$$
\chi(\xi)=\varepsilon(\xi) \xi, \quad \chi_{0}(\xi)=\chi(\xi) /|\xi| .
$$

Here $\varepsilon(\xi)$ is a Dirichlet character modulo the ideal (4) for which $\varepsilon(i)=-i$. For such a character we must have $\varepsilon(1+2 i)= \pm 1$ and, for definiteness, we take

$$
\varepsilon(1+2 i)=-1 \text {. }
$$


Then, for each $r \in N$, $\chi_{0}^{r}$ is a Grössencharacter for the field $K$ and is associated with the ideal (4). Note that $\varepsilon$, when restricted to $Z$, is the non-principal character modulo 4 .

Put

$$
a_{r}(n)=\frac{1}{4} \sum_{|\xi|^{2}=n} \chi^{r}(\xi), \quad \alpha_{r}(n)=a_{r}(n) n^{-r / 2},
$$

and observe that, because of the properties of $\varepsilon$, we have $a_{r}(1)=\alpha_{r}(1)=1$. Let

$$
g_{r}(z)=\sum_{n=1}^{\infty} a_{r}(n) e^{2 \pi i n z} \quad(\operatorname{Im} z>0) .
$$

Then $g_{r}$ is a cusp form of weight $r+1$ and level 64, as noted in [3] (Beispiel 3).

Our object is to obtain estimates for the sums

$$
A(\beta, r ; x)=\sum_{n \leqq x}\left|\alpha_{r}(n)\right|^{2 \beta}
$$

for each $\beta>0$ and $r \in N$ as $x \rightarrow \infty$. In particular, we shall prove

Theorem 1. For any positive integers $N$ and $r$ there exists a positive number $C(N, r)$ such that

$$
A(N, r ; x) \sim C(N, r) x(\log x)^{\delta_{N}}
$$

as $x \rightarrow \infty$, where

$$
\delta_{N}=\left(\begin{array}{c}
2 N-1 \\
N
\end{array}\right)-1 .
$$

To compare this result with (1.1) take $N=2$ and note that $\delta_{2}=2$.

\section{Definitions}

For each prime $p \equiv 1(\bmod 4)$, take any fixed $\xi_{0} \in K$ such that $\left|\xi_{0}\right|^{2}=p$. Then every $\xi$ of norm $p$ is of the form

$$
\xi=\xi_{0} i^{v} \text { or } \xi=\bar{\xi}_{0}(-i)^{v},
$$

where $v=0,1,2,3$ and the bar denotes the complex conjugate. It follows that $\chi(\xi)=\varepsilon\left(\xi_{0}\right) \xi_{0}$ or its conjugate, and so

$$
\alpha_{r}(p)=2 \operatorname{Re}\left\{\varepsilon\left(\xi_{0}\right) \xi_{0} /\left|\xi_{0}\right|\right\}^{r}=2 \cos r \theta_{p},
$$

say, where we may assume that $0 \leqq \theta_{p} \leqq \pi$.

Accordingly, for any prime $p$, we have

$$
\alpha_{r}(p)=2 \cos \theta_{r, p},
$$

where

$$
\theta_{r, p}= \begin{cases}r \theta_{p} & \text { if } p \equiv 1(\bmod 4) \\ \frac{1}{2} \pi & \text { otherwise }\end{cases}
$$


Finally, let

$$
\begin{gathered}
\Psi_{v}(s)=\prod_{p \equiv 1(\bmod 4)}\left(1-2 p^{-s} \cos v \theta_{p}+p^{-2 s}\right)^{-1}, \\
G_{r}(s, \beta)=\sum_{n=1}^{\infty}\left|\alpha_{r}(n)\right|^{2 \beta} n^{-s},
\end{gathered}
$$

the product and series being absolutely convergent for $\sigma=\operatorname{Re} s>1$. We denote by $\boldsymbol{D}$ the set of Dirichlet series holomorphic and nonzero for $\sigma \geqq 1$.

\section{Some lemmas}

Lemma 3.1. For each $r \in N, \alpha_{r}$ is a multiplicative function. Moreover, for any prime $p$ and positive integer $v$

$$
\alpha_{\boldsymbol{r}}\left(p^{v+1}\right)=\alpha_{\boldsymbol{r}}(p) \alpha_{\boldsymbol{r}}\left(p^{v}\right)-\varepsilon(p) \alpha_{\boldsymbol{r}}\left(p^{v-1}\right)
$$

This may be proved exactly as in [6]. It may be remarked that $a_{r}(n)$ is identical with Glaisher's function $\chi_{r}(n)$, which he defined [1] by

$$
\chi_{r}(n)=\frac{1}{2} \sum\left\{(-1)^{(a+b-1) / 2}(a+i b)\right\}^{r},
$$

the summation being taken over all rational integers $a, b$ for which

$$
a^{2}+b^{2}=n, \quad a \text { odd, } b \text { even. }
$$

For $r=4$ and 8 Glaisher used these functions in his work on the representations of a number as a sum of 10 and 18 squares, respectively, and proved their multiplicative properties. In earlier papers he used a slightly different notation that allowed $\chi_{r}(n)$ to be nonzero for even $n$.

From Lemma 3.1 and earlier remarks we deduce

Lemma 3.2. The function $g_{r}(z)$ is a newform of weight $r+1$, level 64 and character $\varepsilon^{r+1}$.

We note that in some ways it is more natural to consider $g_{r}^{*}(z)=g_{r}(z / 8)$. We can then show that $g_{r}^{*}$ has level 8 , weight $r+1$, character $\varepsilon^{r+1}$ and divisor $t=1$, in the notation of [9]. Moreover, $g_{r}^{*}$ can be expressed in terms of derivatives of the simple theta functions. If we write $\psi=\vartheta_{2} \vartheta_{3} \vartheta_{4}$, we find easily from the definition (3.1) that, for any $r=2 m+1$ with $m \geqq 0$,

$$
g_{2 m+1}^{*}(z)=\frac{2^{2 m-1}}{(\pi i)^{m}} \sum_{\mu=0}^{m}\left(\begin{array}{c}
2 n+1 \\
2 \mu
\end{array}\right)(-1)^{\mu} \vartheta_{4}^{(\mu)}(z) \psi^{(n-\mu)}(z),
$$

while, for $r=2 m>0$,

$$
g_{2 m}^{*}(z)=\frac{2^{2 m-1}}{(\pi i)^{m}} \sum_{\mu=0}^{m}\left(\begin{array}{c}
2 m \\
2 \mu
\end{array}\right)(-1)^{u} \vartheta_{3}^{(\mu)}(z) \vartheta_{2}^{(m-\mu)}(z) .
$$

That the expressions on the right-hand sides of (3.2) and (3.3) are in fact cusp forms of weight $r+1$ can be deduced by the methods developed in [7]. Alternatively, 
at any rate when $r$ is divisible by 4 , we can deduce that $g_{r}^{*}$ is a modular form, since in (3.1) $(a+i b)^{r}$ is a spherical harmonic polynomial; see [11].

Lemma 3.3. For each $r \in N, \Psi_{2 r} \in D$. Moreover, for $\sigma \geqq 1$,

$$
G_{r}(s, 1)=\zeta(s) \Psi_{2 r}(s) H_{1}(s)
$$

and

$$
\prod_{p \equiv 1(\bmod 4)}\left(1-p^{-s}\right)^{-2}=\zeta(s) H_{2}(s),
$$

where $H_{1}$ and $H_{2}$ belong to $D$.

Proof. From Lemma 3 of [10] we deduce that $G_{r}(s, 1) / \zeta(s) \in D$ and also that (3.4) holds with

$$
H_{1}(s)=\left(1-2^{-s}\right) L(s, \varepsilon) \prod_{p \equiv 1}\left(1-p^{-2 s}\right),
$$

which is clearly holomorphic and nonzero for $\sigma \geqq 1$; here and later we omit (mod 4) from under product signs, for convenience.

The last part follows, since

$$
\prod_{p \equiv 1}\left(1-p^{-s}\right)^{-2}=\prod_{p \equiv-1}\left(1-p^{-2 s}\right) L(s, \varepsilon) L\left(s, \varepsilon^{2}\right) .
$$

Here the usual $L$-function notation is used.

Lemma 3.4. For fixed $\beta>0$ and $r \in N$,

where

$$
2^{2 \beta-1}|\cos \theta|^{2 \beta}=c_{\beta}+\sum_{n=1}^{\infty} c_{\beta}(n) \cos 2 n \theta \quad(\theta \in \boldsymbol{R}),
$$

$$
c_{\beta}=\frac{2^{2 \beta-1} \Gamma(\beta+1 / 2)}{\sqrt{\pi} \Gamma(\beta+1)},
$$

the Fourier cosine series being uniformly convergent on $\boldsymbol{R}$. If $\beta \in \boldsymbol{N}$, then $c_{\beta}(n)=0$ for $n>\beta$ and

$$
c_{\beta}=\left(\begin{array}{c}
2 \beta-1 \\
\beta
\end{array}\right) .
$$

Proof. The function $|\cos \theta|^{2 \beta}$ has period $\pi$ and is continuous and of bounded variation on $[0, \pi]$.

\section{Proofs of main results}

From (2.2), (2.3) and Lemma 3.1 we deduce that

and that

$$
\alpha_{r}\left(p^{v}\right)=\frac{\sin (v+1) r \theta_{p}}{\sin r \theta_{p}} \text { for } p \equiv 1(\bmod 4), \quad v \in N,
$$

$$
\alpha_{r}\left(p^{2 v}\right)=1, \quad \alpha_{r}\left(p^{2 v-1}\right)=0 \text { for } p \equiv-1(\bmod 4), \quad v \in N
$$


Accordingly, for any $\beta>0$ and $\sigma>1$,

$$
G_{r}(s, \beta)=\prod_{p>2} G_{r}(s, \beta, p),
$$

where, for $p \equiv 1(\bmod 4)$,

$$
G_{r}(s, \beta, p)=1+\left|2 \cos r \theta_{p}\right|^{2 \beta} p^{-s}+\sum_{v=2}^{\infty}\left|\frac{\sin (v+1) r \theta_{p}}{\sin r \theta_{p}}\right|^{2 \beta} p^{-v s}
$$

and, for $p \equiv-1(\bmod 4)$,

$$
G_{r}(s, \beta, p)=\left(1-p^{-2 s}\right)^{-1} .
$$

We are now in a position to prove Theorem 1 . We take a fixed $\beta=N \in N$ and apply Lemma 3.4, so that we have, omitting the suffix $\beta$,

$$
\left|2 \cos r \theta_{p}\right|^{2 N}=2 c+2 \sum_{n=1}^{N} c(n) \cos 2 n r \theta_{p} .
$$

It follows, on using Lemma 2 of [10] and (4.1-3), that

$$
\begin{gathered}
G_{r}(s, \beta)=\prod_{p \equiv-1}\left(1-p^{-2 s}\right)^{-1} \prod_{p \equiv 1}\left(1-p^{-s}\right)^{-2 c} \prod_{n=1}^{N}\left\{\Psi_{2 n r}(s)\right\}^{c(n)} H_{3}(s) \\
=\zeta^{c}(s) H_{4}(s),
\end{gathered}
$$

by Lemma 3.3, with $H_{3}$ and $H_{4}$ in $D$. Theorem 1 now follows from the DelangeIkehara Theorem (see Lemma 4 of [10]), since

$$
c=c_{N}=\left(\begin{array}{c}
2 N-1 \\
N
\end{array}\right)
$$

by Lemma 3.4 .

Theorem 2. For any positive $\varepsilon$ and $\beta$ there exist positive numbers $A_{r}, B_{r}$ depending only on $r, \varepsilon$ and $\beta$ such that, for all sufficiently large $x$,

$$
A_{r} x(\log x)^{\delta(\beta)-\varepsilon} \leqq A(\beta, r ; x) \leqq B_{r} x(\log x)^{\delta(\beta)+\varepsilon},
$$

where $\delta(\beta)=c_{\beta}-1$.

Proof. Take any positive $\varepsilon$ and $\beta$. By Lemma 3.4 we may choose a positive integer $N$ depending on $\varepsilon$ and $\beta$ such that

$$
\left.\left|2^{2 \beta-1}\right| \cos r \theta\right|^{2 \beta}-c_{\beta}-\sum_{n=1}^{N} c_{\beta}(n) \cos 2 n r \theta \mid<\varepsilon
$$

for all real $\theta$. Take a prime $p \equiv 1(\bmod 4)$. We then find that $G_{r}(s, \beta, p)$, regarded as a power series in $p^{-s}$, is majorized by

$$
G_{r}^{+}(s, \beta, p)=1+2\left\{c+\varepsilon+\sum_{n=1}^{N} c(n) \cos 2 n r \theta_{p}\right\} p^{-s}+\sum_{v=2}^{\infty}(v+1)^{2 \beta} p^{-v s}
$$

and 'minorized' by

$$
G_{r}^{-}(s, \beta, p)=1+2\left\{c-\varepsilon+\sum_{n=1}^{N} c(n) \cos 2 n r \theta_{p}\right\} p^{-s} .
$$

Similar arguments to those used to prove Theorem 1 now show that $G_{r}(s, \beta)$ is majorized by $\zeta^{c+\varepsilon}(s) H_{5}(s)$ and minorized by $\zeta^{c-\varepsilon}(s) H_{6}(s)$, where $H_{5}$ and $H_{6}$ belong to $D$. On applying the Delange-Ikehara theorem to these last two Dirichlet series we complete the proof of Theorem 2. It is tempting to conjecture that Theorem 
2 can be replaced by the asymptotic result

$$
A(\beta, r ; x) \sim C x(\log x)^{\delta(\beta)} .
$$

In this connexion it may be noted that, for non-integral $\beta$, Theorem 2 can be replaced by the equivalent result:

$$
A(\beta, r ; x)=x(\log x)^{\delta(\beta)+o(1)},
$$

which is also derivable from a general theorem of Wirsing [13], by using the fact, proved by Hecke [2], that, for $p \equiv 1(\bmod 4)$, the angles $\theta_{p}$ are uniformly distributed in $[0, \pi]$. To remove the term $o(1)$ from the exponent and introduce a constant factor on the right of (4.5) would, however, require more information about the behaviour of the sum

$$
\sum_{p \leqq x, p \equiv 1}\left|\cos r \theta_{p}\right|^{2 \beta}
$$

than is immediately deducible from this fact.

\section{Concluding remarks}

1. In [10] upper (for $\beta>1$ ) and lower (for $0<\beta<1$ ) bounds were obtained for general newforms of any level. The corresponding logarithmic exponent was

$$
\gamma=\gamma(\beta)=2^{2(\beta-1)}-1 \text {. }
$$

It is clear that $\gamma(\beta)>\delta(\beta)$ for $\beta>1$ and $\gamma(\beta)<\delta(\beta)$ for $0<\beta<1$. In particular, $y(2)=3, \delta(2)=2$, as already remarked, and

$$
\gamma(1 / 2)=-1 / 2, \quad \delta(1 / 2)=(2 / \pi)-1 .
$$

2. The methods of [10] can be applied in conjunction with the result (1.1) of Moreno and Shahidi to replace the upper bound given in [10] by

where

$$
\sum_{n \leqq x}\left|\tau_{0}(n)\right|^{2 \beta} \ll x(\log x)^{o(\beta)} \quad(\beta>2),
$$

$$
\varrho(\beta)=2^{2 \beta-3}-1<\gamma(\beta)=2^{2 \beta-2}-1 .
$$

For this purpose one requires the inequality

$$
|2 \cos \theta|^{2 \beta} \leqq 2^{2 \beta} \cos ^{4} \theta=2^{2 \beta-3}(3+4 \cos 2 \theta+\cos 4 \theta) .
$$

In the range $1<\beta<2$, Hölder's inequality gives (5.1) with

$$
\varrho(\beta)=\beta-1 .
$$

3. It is possible that the definitions (1.6) and (3.1) could be used to derive directly, without reference to modular form theory, the results obtained in this paper, but my attempts to do so have been unsuccessful. However, it may be of interest to remark that Titchmarsh's [12] two-dimensional version of van der Corput's method can be directly used to show that

$$
\sum_{n \leqq x} a_{4 r}(n)=O\left(x^{2 r+\alpha+\varepsilon}\right),
$$


where $\alpha=27 / 82$. This is an improvement on the best known bound for cusp form coefficients of weight $1+4 r$, namely

$$
O\left(x^{2 r+1 / 3+\varepsilon}\right) .
$$

No doubt the index $\alpha$ can be decreased slightly.

4. Similar methods can be applied to cusp forms associated with other imaginary quadratic fields and also, for example, to the field $\boldsymbol{Q}(i)$ for the other choice of Dirichlet character in which $(1.5)$ is replaced by $\varepsilon(1+2 i)=1$.

\title{
References
}

[1] Glaisher, J. W. L.: On elliptic-function expansions in which the coefficients are powers of the complex numbers having $n$ as norm. - Quart. J. Pure Appl. Math. 39, 1908, 266300.

[2] Hecke, E.: Eine neue Art von Zetafunktion und ihre Beziehungen zur Verteilung der Primzahlen. II. - Math. Z. 6, 1920, 11-51; Mathematische Werke, Vandenhoek \& Ruprecht, Göttingen, 1959, 249-289.

[3] Hecke, E.: Über Dirichlet-Reihen mit Funktionalgleichung und ihre Nullstellen auf der Mittelgeraden. - S.-B. Math.-Nat. Abt. Bayer. Akad. Wiss. 1937, 73-95; Mathematische Werke, Vandenhoek \& Ruprecht, Göttingen, 1959, 708-730.

[4] Moreno, C. J., and F. Shahidi: The fourth moment of the Ramanujan $\tau$-function. - Math. Ann. 266, 1983, 233-239.

[5] RANKIN, R. A.: Contributions to the theory of Ramanujan's function $\tau(n)$ and similar arithmetical functions. I. The order of the Fourier coefficients of integral modular forms. Proc. Cambridge Philos. Soc. 35, 1939, 357-372.

[6] Rankin, R. A.: A certain class of multiplicative functions. - Duke Math. J. 13, 1946, 281-306.

[7] RANKIN, R. A.: The construction of automorphic forms from the derivatives of a given form. J. Indian Math. Soc. 20, 1956, 103-116.

[8] Rankin, R. A.: An $\Omega$-result for the coefficients of cusp forms. - Math. Ann. 203, 1973, 239250.

[9] Rankin, R. A.: Modular forms and functions. - Cambridge University Press, CambridgeLondon-New York-Melbourne, 1977.

[10] Rankin, R. A.: Sums of powers of cusp form coefficients. - Math. Ann. 263, 1983, 227-236.

[11] Schoeneberg, B.: Das Verhalten von mehrfachen Thetareihen bei Modulsubstitutionen. Math. Ann. 116, 1939, 511-523, 780.

[12] Titchmarsh, E. C.: On van der Corput's method and the zeta-function of Riemann. - Quart. J. Math., Oxford Ser. 2, 1931, 161-173.

[13] Wirsing, E.: Das asymptotische Verhalten von Summen über multiplikative Funktionen. Math. Ann. 143, 1961, 75-102.

\author{
University of Glasgow \\ Department of Mathematics \\ Glasgow G12 8QG \\ Scotland
}

Received 1 September 1983 